Commun. Korean Math. Soc. 25 (2010), No. 1, pp. 83-96

DOI 10.4134/CKMS.2010.25.1.083

\title{
GEODESIC SPHERES AND BALLS OF THE HEISENBERG GROUPS
}

\author{
Changrim Jang, Jihye Park, and Keun Park
}

\begin{abstract}
Let $\mathbb{H}^{2 n+1}$ be the $(2 n+1)$-dimensional Heisenberg group equipped with a left-invariant metric. In this paper we study the Gaussian curvatures of the geodesic spheres and the volumes of geodesic balls in $\mathbb{H}^{2 n+1}$.
\end{abstract}

\section{Introduction}

Let $\mathcal{N}$ be a 2-step nilpotent Lie algebra with an inner product $\langle$,$\rangle and N$ its unique simply connected 2-step nilpotent Lie group with the left invariant metric induced by $\langle$,$\rangle on \mathcal{N}$. Let $\mathcal{Z}$ be the center of $\mathcal{N}$. Then $\mathcal{N}$ is represented by the direct sum of $\mathcal{Z}$ and its orthgonal complement $\mathcal{Z}^{\perp}$.

For each $Z \in \mathcal{Z}$, a skew symmetric linear transformation $j(Z): \mathcal{Z}^{\perp} \rightarrow \mathcal{Z}^{\perp}$ is defined by $j(Z) X=(a d X)^{*} Z$ for $X \in \mathcal{Z}^{\perp}$. Or, equivalently,

$$
\langle j(Z) X, Y\rangle=\langle[X, Y], Z\rangle
$$

for all $X, Y \in \mathcal{Z}^{\perp}$.

A 2-step nilpotent Lie algebra $\mathcal{N}$ is said to be an algebra of Heisenberg type if

$$
j(Z)^{2}=-|Z|^{2} \text { id }
$$

for all $Z \in \mathcal{Z}$. And a Lie group $N$ is said to be a group of Heisenberg type if its Lie algebra $\mathcal{N}$ is of Heisenberg type. The classical Heisenberg groups are examples of Heisenberg type.

The Heisenberg groups are examples of Heisenberg type. That is, let $n \geq 1$ be any integer and $\left\{X_{1}, \ldots, X_{n}, Y_{1}, \ldots, Y_{n}\right\}$ a basis of $R^{2 n}=\mathcal{V}$. Let $\mathcal{Z}$ be an one dimensional vector space spanned by $\{Z\}$. Define

$$
\left[X_{i}, Y_{i}\right]=-\left[Y_{i}, X_{i}\right]=Z
$$

for any $i=1,2, \ldots, n$ with all other brackets are zero. Give on $\mathcal{N}=\mathcal{V} \oplus \mathcal{Z}$ the inner product such that the set of vectors $\left\{X_{i}, Y_{i}, Z \mid i=1,2, \ldots, n\right\}$ forms an

Received February 24, 2009; Revised September 12, 2009.

2000 Mathematics Subject Classification. 53C22, 22E25.

Key words and phrases. Heisenberg group, geodesic sphere, geodesic ball.

This work was supported by 2006 Research Fund of University of Ulsan. 
orthonormal basis. Let $N$ be the simply connected 2-step nilpotent group of Heisenberg type which is determined by $\mathcal{N}$ and equipped with a left-invariant metric induced by the inner product in $\mathcal{N}$. The group $N$ is called the $(2 n+1)$ dimensional Heisenberg group and denoted by $\mathbb{H}^{2 n+1}$.

In this paper, we characterize the Gaussian curvature of the geodesic spheres and the volumes of the geodesic balls on the Heisenberg group $\mathbb{H}^{2 n+1}$ :

Theorem A. Let $0<R<2 \pi$ and $B_{e}(R)$ be the geodesic ball with center $e$ (identity element) and radius $R$ in $\mathbb{H}^{2 n+1}$. Then, the following holds.

$$
\operatorname{Vol}\left(B_{e}(R)\right) \leq \frac{R^{2 n+1}}{2 n+1} \operatorname{Vol}\left(S^{2 n}\right)\left(1+\frac{R^{2}}{12}\right) .
$$

Theorem B. Let $0<R<2 \pi$ and $B_{e}(R)$ be the geodesic ball with center $e$ and radius $R$ in $\mathbb{H}^{3}$. Then, the following holds.

$$
\operatorname{Vol}\left(B_{e}(R)\right)=4 \pi\left(\frac{R^{3}}{3}+2 \sum_{n=2}^{\infty}(-1)^{n} \frac{R^{2 n+1}}{(2 n+1) !(2 n-1)(2 n-3)}\right) .
$$

Theorem C. Let $0<R<2 \pi$ and $S_{e}(R)$ be the geodesic sphere with center $e$ and radius $R$ in $\mathbb{H}^{2 n+1}$. Let $p=\gamma(R) \in S_{e}(R)$, where $\gamma(t)$ is a unit speed geodesic with $\gamma(0)=e$ and $\gamma^{\prime}(0)=X_{0}+Z_{0}$. Then, Gaussian curvature $K(p)$ in $S_{e}(R)$ is given as follows:

$$
\begin{aligned}
K(p)= & \left(-\frac{1}{4}+\frac{\left|Z_{0}\right|^{2}\left(1-\left(1-\left|Z_{0}\right|^{2}\right) \cos \left(\left|Z_{0}\right| R\right)\right)}{2\left(1-\cos \left(\left|Z_{0}\right| R\right)\right)-\left(1-\left|Z_{0}\right|^{2}\right)\left|Z_{0}\right| R \sin \left(\left|Z_{0}\right| R\right)}\right) \\
& \times\left(-\frac{1}{4}\left(\left|Z_{0}\right|^{4}-\left|Z_{0}\right|^{2}+1\right)+\frac{\left|Z_{0}\right|^{2}}{2\left(1-\cos \left(\left|Z_{0}\right| R\right)\right)}\right)^{n-1} .
\end{aligned}
$$

\section{Preliminaries}

Let $\mathcal{N}$ be a 2 -step nilpotent Lie algebra with an inner product $\langle$,$\rangle and N$ be its unique simply connected 2 -step nilpotent Lie group with the left invariant metric induced by $\langle$,$\rangle on \mathcal{N}$. The center of $\mathcal{N}$ is denoted by $\mathcal{Z}$. Then $\mathcal{N}$ can be expressed as the direct sum of $\mathcal{Z}$ and its orthogonal complement $\mathcal{Z}^{\perp}$.

Recall that for $Z \in \mathcal{Z}$, a skew symmetric linear transformation $j(Z): \mathcal{Z}^{\perp} \rightarrow$ $\mathcal{Z}^{\perp}$ is defined by $j(Z) X=(\operatorname{ad} X)^{*} Z$ for $X \in \mathcal{Z}^{\perp}$. Or, equivalently,

$$
\langle j(Z) X, Y\rangle=\langle[X, Y], Z\rangle
$$

for $X, Y \in \mathcal{Z}^{\perp}$. A 2-step nilpotent Lie group $N$ is said to be of Heisenberg type if

$$
j(Z)^{2}=-|Z|^{2} \text { id }
$$

for all $Z \in \mathcal{Z}$. 
Let $\nabla$ be the unique Riemannian connection on $\mathcal{N}$. If $\xi_{1}, \xi_{2}$ and $\xi_{3}$ are left-invariant vector fields, then the formula of the covariant derivative

$$
\begin{aligned}
\left\langle\xi_{3}, \nabla_{\xi_{1}} \xi_{2}\right\rangle=\frac{1}{2}\{ & \xi_{1}\left\langle\xi_{2}, \xi_{3}\right\rangle+\left\langle\xi_{1},\left[\xi_{3}, \xi_{2}\right]\right\rangle+\xi_{2}\left\langle\xi_{1}, \xi_{3}\right\rangle \\
& \left.+\left\langle\xi_{2},\left[\xi_{3}, \xi_{1}\right]\right\rangle-\xi_{3}\left\langle\xi_{2}, \xi_{1}\right\rangle-\left\langle\xi_{3},\left[\xi_{2}, \xi_{1}\right]\right\rangle\right\}
\end{aligned}
$$

can be reduced to

$$
\left\langle\xi_{3}, \nabla_{\xi_{1}} \xi_{2}\right\rangle=\frac{1}{2}\left\{\left\langle\xi_{1},\left[\xi_{3}, \xi_{2}\right]\right\rangle+\left\langle\xi_{2},\left[\xi_{3}, \xi_{1}\right]\right\rangle-\left\langle\xi_{3},\left[\xi_{2}, \xi_{1}\right]\right\rangle\right\} .
$$

Using this, the covariant derivatives on $\mathcal{N}$ are given as follows:

Lemma 2.1 ([3]). For a 2-step nilpotent Lie group $N$ with a left invariant metric, the following hold.

(1) $\nabla_{X} Y=\frac{1}{2}[X, Y]$ for $X, Y \in \mathcal{Z}^{\perp}$.

(2) $\nabla_{X} Z=\nabla_{Z} X=-\frac{1}{2} j(Z) X$ for $X \in \mathcal{Z}^{\perp}$ and $Z \in \mathcal{Z}$.

(3) $\nabla_{Z} Z^{*}=0$ for $Z, Z^{*} \in \mathcal{Z}$.

Let $\gamma(t)$ be a curve in $N$ such that $\gamma(0)=e$ (identity element in $N$ ) and $\gamma^{\prime}(0)=X_{0}+Z_{0}$, where $X_{0} \in \mathcal{Z}^{\perp}$ and $Z_{0} \in \mathcal{Z}$. Since $\exp : \mathcal{N} \rightarrow N$ is a diffeomorphism $([10])$, the curve $\gamma(t)$ can be expressed uniquely by $\gamma(t)=$ $\exp (X(t)+Z(t)]$ with

$$
\begin{array}{rll}
X(t) \in \mathcal{Z}^{\perp}, & X^{\prime}(0)=X_{0}, & X(0)=0 \\
Z(t) \in \mathcal{Z}, & Z^{\prime}(0)=Z_{0}, & Z(0)=0 .
\end{array}
$$

A. Kaplan ([7]) shows that the curve $\gamma(t)$ is a geodesic in $N$ if and only if

$$
\begin{array}{r}
X^{\prime \prime}(t)=j\left(Z_{0}\right) X^{\prime}(t), \\
Z^{\prime}(t)+\frac{1}{2}\left[X^{\prime}(t), X(t)\right] \equiv Z_{0} .
\end{array}
$$

The following lemma is useful in the later.

Lemma 2.2 ([3]). Let $N$ be a simply connected 2-step nilpotent Lie group with a left invariant metric, and let $\gamma(t)$ be a geodesic of $N$ with $\gamma(0)=e$ and $\gamma^{\prime}(0)=X_{0}+Z_{0}$, where $X_{0} \in \mathcal{Z}^{\perp}$ and $Z_{0} \in \mathcal{Z}$. Then, one has

$$
\gamma^{\prime}(t)=d l_{\gamma(t)}\left(X^{\prime}(t)+Z_{0}\right), t \in R,
$$

where $X^{\prime}(t)=e^{t j\left(Z_{0}\right)} X_{0}$ and $l_{\gamma(t)}$ is the left translation by $\gamma(t)$.

Throughout this paper, different tangent spaces will be identified with $\mathcal{N}$ via left translation. So, in above lemma, we can consider $\gamma^{\prime}(t)$ as

$$
\gamma^{\prime}(t)=X^{\prime}(t)+Z_{0}=e^{t j\left(Z_{0}\right)} X_{0}+Z_{0} .
$$




\section{Main results}

Let $\mathbb{H}^{2 n+1}$ be the $(2 n+1)$-dimensional Heisenberg group with a left invariant metric and $\mathcal{N}$ its Lie algebra. Let $\gamma(t)$ be a unit speed geodesic on $\mathbb{H}^{2 n+1}$ with $\gamma(0)=e$ (the identity element of $\mathbb{H}^{2 n+1}$ ) and $\gamma^{\prime}(0)=X_{0}+Z_{0}$, where $X_{0} \in \mathcal{Z}^{\perp}$ and $Z_{0} \in \mathcal{Z}$. Assume that $X_{0} \neq 0$ and $Z_{0} \neq 0$. Since

$$
\left\{X_{0}+Z_{0}, \frac{\left|Z_{0}\right|}{\left|X_{0}\right|} X_{0}-\frac{\left|X_{0}\right|}{\left|Z_{0}\right|} Z_{0}, \frac{1}{\left|Z_{0}\right|\left|X_{0}\right|} j\left(Z_{0}\right) X_{0}\right\}
$$

is an ortonormal set in $\mathcal{N}$, we can obtain an orthonormal basis

$$
\begin{gathered}
\mathcal{B}=\left\{X_{0}+Z_{0}, \frac{\left|Z_{0}\right|}{\left|X_{0}\right|} X_{0}-\frac{\left|X_{0}\right|}{\left|Z_{0}\right|} Z_{0}, \frac{1}{\left|Z_{0}\right|\left|X_{0}\right|} j\left(Z_{0}\right) X_{0}, Y_{k}, \frac{1}{\left|Z_{0}\right|} j\left(Z_{0}\right) Y_{k} \mid Y_{k} \in \mathcal{Z}^{\perp},\right. \\
k=1,2, \ldots, n-1\}
\end{gathered}
$$

by adding

$$
\left\{Y_{k}, \frac{1}{\left|Z_{0}\right|} j\left(Z_{0}\right) Y_{k} \mid Y_{k} \in \mathcal{Z}^{\perp}, k=1,2, \ldots, n-1\right\}
$$

to

$$
\left\{X_{0}+Z_{0}, \frac{\left|Z_{0}\right|}{\left|X_{0}\right|} X_{0}-\frac{\left|X_{0}\right|}{\left|Z_{0}\right|} Z_{0}, \frac{1}{\left|Z_{0}\right|\left|X_{0}\right|} j\left(Z_{0}\right) X_{0}\right\}
$$

Then, it is easy to show that $\left[X_{0}, Y_{k}\right]=\left[X_{0}, j\left(Z_{0}\right) Y_{k}\right]=0$ for each $k=$ $1,2, \ldots, n-1$.

Proposition 3.1 ([1], [6]). Assume that $X_{0} \neq 0$ and $Z_{0} \neq 0$. If $J(t)$ is a normal Jacobi field along $\gamma$ in $\mathbb{H}^{2 n+1}$ with $J(0)=0$, then

$$
\begin{aligned}
J(t)= & \left(c_{1}\left(\sin \left(\left|Z_{0}\right| t\right)-\left(1-\left|Z_{0}\right|^{2}\right)\left|Z_{0}\right| t\right)+c_{2}\left(1-\cos \left(\left|Z_{0}\right| t\right)\right)\right) e_{1}(t) \\
& +\left(c_{1}\left|Z_{0}\right|\left(\cos \left(\left|Z_{0}\right| t\right)-1\right)+c_{2}\left|Z_{0}\right| \sin \left(\left|Z_{0}\right| t\right) e_{2}(t)\right. \\
& +\sum_{k=2}^{n}\left[\left\{\frac{c_{2 k-1}}{\left|Z_{0}\right|} \sin \left(\left|Z_{0}\right| t\right)+\frac{c_{2 k}}{\left|Z_{0}\right|}\left(1-\cos \left(\left|Z_{0}\right| t\right)\right)\right\} e_{2 k-1}(t)\right. \\
& \left.\quad+\left\{c_{2 k-1}\left|Z_{0}\right|\left(\cos \left(\left|Z_{0}\right| t\right)-1\right)+c_{2 k}\left|Z_{0}\right| \sin \left(\left|Z_{0}\right| t\right)\right\} e_{2 k}(t)\right],
\end{aligned}
$$

where $c_{2 k-1}, c_{2 k}, k=1,2, \ldots, n$ are arbitrary constants and $e_{2 k-1}(t), e_{2 k}(t), k=$ $1,2, \ldots, n$ are given in Lemma 3.2.

Lemma 3.2. Assume that $X_{0} \neq 0$ and $Z_{0} \neq 0$. Let

$$
\begin{aligned}
& e_{1}(t)=\frac{\left|Z_{0}\right|}{\left|X_{0}\right|} X^{\prime}(t)-\frac{\left|X_{0}\right|}{\left|Z_{0}\right|} Z_{0}, \\
& e_{2}(t)=\frac{1}{\left|Z_{0}\right|\left|X_{0}\right|} j\left(Z_{0}\right) X^{\prime}(t)
\end{aligned}
$$

and let

$$
\begin{aligned}
& e_{2 k-1}(t)=e^{t j\left(Z_{0}\right)} Y_{k}, \\
& e_{2 k}(t)=\frac{1}{\left|Z_{0}\right|} e^{t j\left(Z_{0}\right)} j\left(Z_{0}\right) Y_{k} \quad \text { for each } k=2,3, \ldots, n .
\end{aligned}
$$


Then, $\left\{\gamma^{\prime}(t), e_{2 k-1}(t), e_{2 k}(t) \mid k=1,2, \ldots, n\right\}$ is an orthonormal frame along $\gamma(t)$ on $\mathbb{H}^{2 n+1}$ such that

(1) $\nabla_{\gamma^{\prime}(t)} e_{1}(t)=\frac{1}{2} e_{2}(t)$ and $\nabla_{\gamma^{\prime}(t)} e_{2}(t)=-\frac{1}{2} e_{1}(t)$

(2) $\nabla_{\gamma^{\prime}(t)} e_{2 k-1}(t)=\frac{\left|Z_{0}\right|}{2} e_{2 k}(t)$ and $\nabla_{\gamma^{\prime}(t)} e_{2 k}(t)=-\frac{\left|Z_{0}\right|}{2} e_{2 k-1}(t)$ for each $k=2,3, \ldots, n$.

Or simply,

$$
\left[\begin{array}{l}
e_{1}^{\prime}(t) \\
e_{2}^{\prime}(t)
\end{array}\right]=\frac{1}{2}\left[\begin{array}{cc}
0 & 1 \\
-1 & 0
\end{array}\right]\left[\begin{array}{l}
e_{1}(t) \\
e_{2}(t)
\end{array}\right]
$$

and

$$
\left[\begin{array}{c}
e_{2 k-1}^{\prime}(t) \\
e_{2 k}^{\prime}(t)
\end{array}\right]=\frac{\left|Z_{0}\right|}{2}\left[\begin{array}{cc}
0 & 1 \\
-1 & 0
\end{array}\right]\left[\begin{array}{c}
e_{2 k-1}(t) \\
e_{2 k}(t)
\end{array}\right]
$$

for $k=2,3, \ldots, n$.

The following proposition is a slight modification of Proposition 3.1, which is useful.

Proposition 3.3. For each $k=1,2, \ldots, n$, let $J_{2 k-1}(t)$ and $J_{2 k}(t)$ be the Jacobi fields with $J_{2 k-1}(0)=J_{2 k}(0)=0, J_{2 k-1}^{\prime}(0)=e_{2 k-1}(0)$ and $J_{2 k}^{\prime}(0)=$ $e_{2 k}(0)$. Then, we have that

(1) for $k=1$,

$$
\left[\begin{array}{l}
J_{1}(t) \\
J_{2}(t)
\end{array}\right]=B_{1}(t)\left[\begin{array}{l}
e_{1}(t) \\
e_{2}(t)
\end{array}\right]
$$

where

$$
B_{1}(t)=\frac{1}{\left|Z_{0}\right|^{3}}\left[\begin{array}{cc}
\sin \left(\left|Z_{0}\right| t\right)-\left(1-\left|Z_{0}\right|^{2}\right)\left|Z_{0}\right| t & \left|Z_{0}\right|\left(\cos \left(\left|Z_{0}\right| t\right)-1\right) \\
\left|Z_{0}\right|\left(1-\cos \left(\left|Z_{0}\right| t\right)\right) & \left|Z_{0}\right|^{2} \sin \left(\left|Z_{0}\right| t\right)
\end{array}\right]
$$

(2) for $k=2,3, \ldots, n$

$$
\left[\begin{array}{c}
J_{2 k-1}(t) \\
J_{2 k}(t)
\end{array}\right]=B_{k}(t)\left[\begin{array}{c}
e_{2 k-1}(t) \\
e_{2 k}(t)
\end{array}\right]
$$

where

$$
B_{k}(t)=\left[\begin{array}{cc}
\frac{1}{\left|Z_{0}\right|} \sin \left(\left|Z_{0}\right| t\right) & \left|Z_{0}\right|\left(\cos \left(\left|Z_{0}\right| t\right)-1\right) \\
\frac{1}{\left|Z_{0}\right|^{3}}\left(1-\cos \left(\left|Z_{0}\right| t\right)\right) & \frac{1}{\left|Z_{0}\right|} \sin \left(\left|Z_{0}\right| t\right)
\end{array}\right] .
$$

Proof. Let $J(t)$ be a normal Jacobi field along $\gamma(t)$ with $J(0)=0$. Then, by Proposition 3.1 and Lemma 3.2, we can represent $J(t)$ as follow.

$$
J(t)=\left[\begin{array}{lllll}
c_{1} & c_{2} & \cdots & c_{2 n-1} & c_{2 n}
\end{array}\right]\left[\begin{array}{cccc}
B_{1}(t) & 0 & \cdots & 0 \\
0 & B_{2}(t) & \cdots & 0 \\
\vdots & \vdots & \ddots & \vdots \\
0 & 0 & \cdots & B_{n}(t)
\end{array}\right]\left[\begin{array}{c}
e_{1}(t) \\
e_{2}(t) \\
\vdots \\
e_{2 n-1}(t) \\
e_{2 n}(t)
\end{array}\right]
$$


Since $B_{k}(0)=0$ and $B_{k}^{\prime}(0)=I$ for each $k=1,2, \ldots, n$, we have that

$$
J^{\prime}(0)=c_{l} e_{1}(0)+c_{2} e_{2}(0)+\cdots+c_{2 n-1} e_{2 n-1}(0)+c_{2 n} e_{2 n}(0) .
$$

Just letting $J^{\prime}(0)=e_{k}(0)$ for each $k=1,2, \ldots, 2 n$, we completes the proof.

Corollary 3.4 ([1], [6]). Let $\mathbb{H}^{2 n+1}$ be the $(2 n+1)$-dimensional Heisenberg group and $\mathcal{N}$ its Lie algebra. Let $\gamma(t)$ be a unit speed geodesic in $N$ with $\gamma(0)=e$ (the identity element of $N)$ and $\gamma^{\prime}(0)=X_{0}+Z_{0}$, where $X_{0} \in \mathcal{Z}^{\perp}$ and $Z_{0} \in \mathcal{Z}$.

(1) If $Z_{0} \neq 0$, then all the conjugate points along $\gamma$ are at $t \in \frac{2 \pi}{\left|Z_{0}\right|} \mathbb{Z}^{*} \cup \mathbb{A}$ where

$$
\mathbb{Z}^{*}=\{ \pm 1, \pm 2, \ldots\}
$$

and

$$
\mathbb{A}=\left\{t \in \mathbb{R}-\{0\} \mid\left(1-\left|Z_{0}\right|^{2}\right) \frac{\left|Z_{0}\right| t}{2}=\tan \frac{\left|Z_{0}\right| t}{2}\right\} .
$$

In particular, $\frac{2 \pi}{\left|Z_{0}\right|}$ is the first conjugate point of e along $\gamma$.

(2) If $Z_{0}=0$, then there are no conjugate points along $\gamma$.

G. Walschap ([11]) showed that the first conjugate loci and the cut loci are equal in the case of the groups of Heisenberg type or the 2-step nilpotent groups with one-dimensional center.

So, we see that the geodesic sphere $S_{e}(r)$ with center $e$ and radius $r$ is defined if and only if $r \leq 2 \pi$. So, we consider the geodesic spheres $S_{e}(r)$ and the geodesic balls $B_{e}(r)$ with the radius $r \leq 2 \pi$.

Note that

$$
\operatorname{det}\left(B_{1}(t)\right)=\frac{1}{\left|Z_{0}\right|^{4}}\left\{2\left(1-\cos \left(\left|Z_{0}\right| t\right)\right)-\left(1-\left|Z_{0}\right|^{2}\right)\left|Z_{0}\right| t \sin \left(\left|Z_{0}\right| t\right)\right\}
$$

and

$$
\operatorname{det}\left(B_{k}(t)\right)=\frac{2}{\left|Z_{0}\right|^{2}}\left(1-\cos \left(\left|Z_{0}\right| t\right)\right)
$$

for each $k=2,3, \ldots, n$.

Lemma 3.5 ([9]). For $t \geq 0$, the following holds.

$$
\operatorname{det}\left(B_{1}(t)\right)=\frac{1}{\left|Z_{0}\right|^{4}}\left\{2\left(1-\cos \left(\left|Z_{0}\right| t\right)\right)-\left(1-\left|Z_{0}\right|^{2}\right)\left|Z_{0}\right| t \sin \left(\left|Z_{0}\right| t\right)\right\} \geq 0 .
$$

Lemma 3.6. For $x>0$, the followings are hold.

(1) $\frac{\sin x}{x} \leq 1$.

(2) $\frac{1-\cos x}{x^{2}} \leq \frac{1}{2}$.

(3) $\frac{2(1-\cos x)-x \sin x}{x^{4}} \leq \frac{1}{12}$. 
Proof. We give only the proof of (3) since others are easy. Let

$$
f(t)=\frac{1}{12} x^{4}-\{2(1-\cos x)-x \sin x\} .
$$

Then, we have that

$$
f^{\prime}(x)=\frac{1}{3} x^{3}-(\sin x-x \cos x)
$$

and

$$
f^{\prime \prime}(x)=x(x-\sin x)
$$

Since $f^{\prime \prime}(x)>0$ for $x>0$ and $f^{\prime}(0)=0$, we see that $f^{\prime}(x)>0$. Since $f(0)=0$, we get that $f(x)>0$ for $x>0$.

Let $M$ be a Riemannian manifold with a metric $g$ and $p \in M$. Take an orthonormal basis $\left\{u_{1}, u_{2}, \ldots, u_{n}\right\}$ of $T_{p} M$ and let $\left(x_{1}, x_{2}, \ldots, x_{n}\right)$ be the coordinates determined by $\left\{u_{1}, u_{2}, \ldots, u_{n}\right\}$. This local coordinate system is called the normal coordinate system at $p$. It is easy to show that

$$
\frac{\partial}{\partial x_{i m}}=\left(d \exp _{p}\right)_{\sum_{i=1}^{n} x_{i} u_{i}}\left(u_{i}\right)
$$

where $m=\exp _{p}\left(\sum_{i=1}^{n} x_{i} u_{i}\right)$. Then, the volume form $v_{g}$ on $U_{p}$ is given by

$$
v_{g}=\sqrt{\operatorname{det}\left(g\left(\frac{\partial}{\partial x_{i}}, \frac{\partial}{\partial x_{j}}\right)\right)} d x_{1} \wedge d x_{2} \wedge \cdots \wedge d x_{n},
$$

where $g_{i j}$ is the metric coefficients of $g$ in $U_{p}$. Therefore, the volume of the geodesic ball $B_{p}(r)$ is given by

$$
\operatorname{Vol}\left(B_{p}(r)\right)=\int_{\exp _{p}^{-1}\left(B_{p}(r)\right)} \exp _{p}^{*} v_{g} .
$$

Let $\gamma(t)$ be the unit speed geodesic in $M$ with $\gamma(0)=p, \gamma^{\prime}(0)=u_{1}$ and let $J_{i}(t)$ be the Jacobi field with $J_{i}(0)=0$ and $J_{i}^{\prime}(0)=u_{i}$ for each $i=2,3, \ldots, n$. Then we know that

$$
\left(d \exp _{p}\right)_{t u_{1}} u_{1}=\gamma^{\prime}(t)
$$

and

$$
\left(d \exp _{p}\right)_{t u_{1}} u_{i}=\frac{1}{t} J_{i}(t)
$$

for each $i=2,3, \ldots, n$. So, we see that

$$
\sqrt{\operatorname{det}\left(g\left(\frac{\partial}{\partial x_{i}}, \frac{\partial}{\partial x_{j}}\right)\right)}=t^{-(n-1)} \sqrt{\operatorname{det}\left(g\left(J_{i}(t), J_{j}(t)\right)\right)} .
$$

Hence, we have that

$$
\begin{aligned}
\exp _{p}^{*} v_{g} & =t^{-(n-1)} \sqrt{\operatorname{det}\left(g\left(J_{i}(t), J_{j}(t)\right)\right)} d x_{1} d x_{2} \cdots d x_{n} \\
& =\sqrt{\operatorname{det}\left(g\left(J_{i}(t), J_{j}(t)\right)\right)} d t d u
\end{aligned}
$$


where $d u$ denote the canonical measure of the unit sphere $S^{n-1}$. Therefore, by Fubini's Theorem we get that

$$
\operatorname{Vol}\left(B_{p}(r)\right)=\int_{S^{n-1}} \int_{0}^{r} \sqrt{\operatorname{det}\left(g\left(J_{i}(t), J_{j}(t)\right)\right)} d t d u .
$$

Now we are ready to prove the following proposition which is concerned to the volume of geodesic ball in the Heisenberg group $\mathbb{H}^{2 n+1}$ with a left invariant metric.

Theorem 3.7. Let $0<R<2 \pi$ and $B_{e}(R)$ be the geodesic ball with center $e$ and radius $R$ in $\mathbb{H}^{2 n+1}$. Then, the following holds.

$$
\operatorname{Vol}\left(B_{e}(R)\right) \leq \frac{R^{2 n+1}}{2 n+1} \operatorname{Vol}\left(S^{2 n}\right)\left(1+\frac{R^{2}}{12}\right) .
$$

Proof. Using Propsition 3.3, we obtain that

$$
\begin{aligned}
& \operatorname{det}\left(\left\langle J_{i}(t), J_{j}(t)\right\rangle\right) \\
& =\operatorname{det}\left(J_{i}(t) \cdot J_{j}(t)\right) \\
& =\operatorname{det}\left(\left[\begin{array}{c}
J_{1}(t) \\
J_{2}(t) \\
\vdots \\
J_{2 n-1}(t) \\
J_{2 n}(t)
\end{array}\right]\left[\begin{array}{lllll}
J_{1}(t) & J_{2}(t) & \cdots & J_{2 n-1}(t) & J_{2 n}(t)
\end{array}\right]\right) \\
& =\prod_{k=1}^{n} \operatorname{det}\left(B_{k}(t)\left[\begin{array}{c}
e_{2 k-1}(t) \\
e_{2 k}(t)
\end{array}\right] \cdot t\left(B_{k}(t)\left[\begin{array}{c}
e_{2 k-1}(t) \\
e_{2 k}(t)
\end{array}\right]\right)\right) \\
& =\prod_{k=1}^{n} \operatorname{det}\left(B_{k}(t) \cdot{ }^{t}\left(B_{k}(t)\right)\right) \\
& =\left(\frac{1}{\left|Z_{0}\right|^{4}}\left\{2\left(1-\cos \left(\left|Z_{0}\right| t\right)\right)-\left(1-\left|Z_{0}\right|^{2}\right)\left|Z_{0}\right| t \sin \left(\left|Z_{0}\right| t\right)\right\}\left\{\frac{2}{\left|Z_{0}\right|^{2}}\left(1-\cos \left(\left|Z_{0}\right| t\right)\right)\right\}^{n-1}\right)^{2} \text {. }
\end{aligned}
$$

So, by Lemma 3.5 and Lemma 3.6, we have that

$$
\begin{aligned}
& \sqrt{\left.\operatorname{det}\left(\left\langle J_{i}(t), J_{j}(t)\right\rangle\right)\right)} \\
= & \frac{1}{\left|Z_{0}\right|^{4}}\left\{2\left(1-\cos \left(\left|Z_{0}\right| t\right)\right)-\left(1-\left|Z_{0}\right|^{2}\right)\left|Z_{0}\right| t \sin \left(\left|Z_{0}\right| t\right)\right\}\left\{\frac{2}{\left|Z_{0}\right|^{2}}\left(1-\cos \left(\left|Z_{0}\right| t\right)\right)\right\}^{n-1} \\
= & \left\{\frac{2\left(1-\cos \left(\left|Z_{0}\right| t\right)\right)-\left|Z_{0}\right| t \sin \left(\left|Z_{0}\right| t\right)}{\left(\left|Z_{0}\right| t\right)^{4}} t^{4}+\frac{\sin \left(\left|Z_{0}\right| t\right)}{\left|Z_{0}\right| t} t^{2}\right\}\left\{\frac{2\left(1-\cos \left(\left|Z_{0}\right| t\right)\right)}{\left(\left|Z_{0}\right| t\right)^{2}} t^{2}\right\}^{n-1} \\
\leq & \left(\frac{1}{12} t^{4}+t^{2}\right) t^{2 n-2} .
\end{aligned}
$$


Hence, we get that

$$
\begin{aligned}
\operatorname{Vol}\left(B_{e}(R)\right) & =\int_{S^{2 n}} \int_{0}^{R} \sqrt{\operatorname{det}\left(\left\langle J_{i}(t), J_{j}(t)\right\rangle\right)} d t d u \\
& \leq \operatorname{Vol}\left(S^{2 n}\right) \int_{0}^{R}\left(\frac{1}{12} t^{4}+t^{2}\right) t^{2 n-2} d t \\
& =\operatorname{Vol}\left(S^{2 n}\right)\left(\frac{R^{2 n+3}}{12(2 n+3)}+\frac{R^{2 n+1}}{2 n+1}\right) \\
& =\frac{R^{2 n+1}}{2 n+1} \operatorname{Vol}\left(S^{2 n}\right)\left(1+\frac{2 n+1}{12(2 n+3)} R^{2}\right) \\
& \leq \frac{R^{2 n+1}}{2 n+1} \operatorname{Vol}\left(S^{2 n}\right)\left(1+\frac{R^{2}}{12}\right) .
\end{aligned}
$$

This completes the proof.

Theorem 3.8. Let $0<R<2 \pi$ and $B_{e}(R)$ be the geodesic ball with center $e$ and radius $R$ in $\mathbb{H}^{3}$. Then, the following holds.

$$
\operatorname{Vol}\left(B_{e}(R)\right)=4 \pi\left(\frac{R^{3}}{3}+2 \sum_{n=2}^{\infty}(-1)^{n} \frac{R^{2 n+1}}{(2 n+1) !(2 n-1)(2 n-3)}\right) .
$$

Proof. Let $u=\left(x_{1}, x_{2}, x_{3}\right) \in S^{2}$ and

$$
\begin{aligned}
f\left(x_{3}, t\right) & =\sqrt{\operatorname{det}\left(\left\langle J_{i}(t), J_{j}(t)\right\rangle\right)} \\
& =\frac{2\left(1-\cos \left(x_{3} t\right)\right)-x_{3} t \sin \left(x_{3} t\right)}{\left(x_{3} t\right)^{4}} t^{4}+\frac{\sin \left(x_{3} t\right)}{x_{3} t} t^{2} .
\end{aligned}
$$

Then, we see that

$$
\operatorname{Vol}\left(B_{e}(R)\right)=\int_{S^{2}} \int_{0}^{R} f\left(x_{3}, t\right) d t d u .
$$

Let $D=\left\{\left(x_{1}, x_{2}\right) \mid x_{1}^{2}+x_{2}^{2} \leq 1\right\}$, then since area element $d u$ on the sphere $S^{2}$ is given by

$$
d u=\frac{1}{\sqrt{1-\left(x_{1}^{2}+x_{2}^{2}\right)}} d x_{1} d x_{2}
$$

we have that

$$
\operatorname{Vol}\left(B_{e}(R)\right)=2 \int_{D} \int_{0}^{R} f\left(\sqrt{1-\left(x_{1}^{2}+x_{2}^{2}\right)}, t\right) \frac{1}{\sqrt{1-\left(x_{1}^{2}+x_{2}^{2}\right)}} d t d x_{1} d x_{2} .
$$

Changing the coordinates on $D$ to polar coordinates, we have

$$
\operatorname{Vol}\left(B_{e}(R)\right)=4 \pi \int_{0}^{1} \int_{0}^{R} f\left(\sqrt{1-r^{2}}, t\right) \frac{r}{\sqrt{1-r^{2}}} d t d r .
$$


Replacing $x=\sqrt{1-r^{2}}$, we see that

$$
\operatorname{Vol}\left(B_{e}(R)\right)=4 \pi \int_{0}^{1} \int_{0}^{R} f(x, t) d t d x
$$

where

$$
f(x, t)=\frac{2(1-\cos (x t))-x t \sin (x t)}{(x t)^{4}} t^{4}+\frac{\sin (x t)}{x t} t^{2}
$$

Since

$$
\frac{\sin (x t)}{x t}=\sum_{n=1}^{\infty}(-1)^{n-1} \frac{(x t)^{2 n-2}}{(2 n-1) !}
$$

and

$$
\begin{aligned}
& \frac{2(1-\cos (x t))-x t \sin (x t)}{(x t)^{4}} \\
= & \frac{1}{(x t)^{4}}\left(2 \sum_{n=1}^{\infty}(-1)^{n-1} \frac{(x t)^{2 n}}{(2 n) !}-x t \sum_{n=1}^{\infty}(-1)^{n-1} \frac{(x t)^{2 n-1}}{(2 n-1) !}\right) \\
= & \sum_{n=2}^{\infty}(-1)^{n-1}\left(\frac{2}{(2 n) !}-\frac{1}{(2 n-1) !}\right)(x t)^{2 n-4},
\end{aligned}
$$

we see that

$$
\int_{0}^{1} \frac{\sin (x t)}{x t} d x=\sum_{n=1}^{\infty}(-1)^{n-1} \frac{t^{2 n-2}}{(2 n-1) !(2 n-1)}
$$

and

$$
\int_{0}^{1} \frac{2(1-\cos (x t))-x t \sin (x t)}{(x t)^{4}} d x=\sum_{n=2}^{\infty} \frac{(-1)^{n-1}}{2 n-3}\left(\frac{2}{(2 n) !}-\frac{1}{(2 n-1) !}\right) t^{2 n-4} .
$$

Hence, we have that

$$
\begin{aligned}
& \int_{0}^{1} f(x, t) d x \\
= & t^{4} \int_{0}^{1} \frac{2(1-\cos (x t))-x t \sin (x t)}{(x t)^{4}} d x+t^{2} \int_{0}^{1} \frac{\sin (x t)}{x t} d x \\
= & 2 \sum_{n=1}^{\infty} \frac{(-1)^{n}}{(2 n) !(2 n-1)(2 n-3)} t^{2 n}
\end{aligned}
$$

Therefore, we see that

$$
\begin{aligned}
\operatorname{Vol}\left(B_{e}(R)\right) & =4 \pi \int_{0}^{R} \int_{0}^{1} f(x, t) d x d t \\
& =8 \pi \sum_{n=1}^{\infty} \frac{(-1)^{n}}{(2 n+1) !(2 n-1)(2 n-3)} R^{2 n+1} .
\end{aligned}
$$


Or,

$$
\operatorname{Vol}\left(B_{e}(R)\right)=4 \pi\left(\frac{R^{3}}{3}+2 \sum_{n=2}^{\infty} \frac{(-1)^{n}}{(2 n+1) !(2 n-1)(2 n-3)} R^{2 n+1}\right) .
$$

Let $\bar{M}$ be a Riemannian manifold, $M$ its Riemannian submanifold with codimension 1, $p \in M$ and a normal vector $\eta$ to $T_{p}(M)$. The shape operator

$$
S_{p}: T_{p}(M) \rightarrow T_{p}(M)
$$

is defined by

$$
S_{p}(x)=-\left(\bar{\nabla}_{x} N\right)^{T} \text { for any } x \in T_{p}(M),
$$

where $N$ is a local extension of $\eta$ normal to $M$ and ${ }^{T}$ denotes the tangential component to $T_{p}(M)$. It is easy to show that if $\eta$ and its extension $N$ are unit vector and unit vector field, then the shape operator is given by

$$
S_{p}(x)=-\bar{\nabla}_{x} N \text { for any } x \in T_{p}(M) .
$$

The shape operator $S_{p}$ is symmetric, so there exists an orthonormal basis of eigenvectors $\left\{e_{1}, e_{2}, \ldots, e_{n}\right\}$ with real eigenvalues $\lambda_{1}, \lambda_{2}, \ldots, \lambda_{n}$. We say that the $e_{i}$ are principal directions and $\lambda_{i}$ are principal curvatures of $M$ at $p$. The determinant of shape operator

$$
\operatorname{det}\left(S_{p}\right)=\lambda_{1} \times \lambda_{2} \times \cdots \times \lambda_{n}
$$

is called the Gaussian curvature.

Lemma 3.9 ([12]). Let $\bar{M}$ be a Riemannian manifold, $m \in \bar{M}$ and $M$ the geodesic sphere with center $m$ and radius $r>0$ and $\gamma(t)$ be a unit speed geodesic with $\gamma(0)=m$. Let $J(t)$ be a Jacobi vector fields along $\gamma$ with $J(0)=0$, which is normal to $\gamma$ and $S_{p}$ the shape operator of $M$ at $p=\gamma(r)$. Then, we have that $S_{p}(J(r))=-J^{\prime}(r)$.

Using this lemma, we characterize the Gaussian curvatures on the geodesic spheres of the Heisenberg group $\mathbb{H}^{2 n+1}$.

Theorem 3.10. Let $0<R<2 \pi$ and $S_{e}(R)$ be the geodesic sphere with center $e$ and radius $R$ in $\mathbb{H}^{2 n+1}$. Let $p=\gamma(R) \in S_{e}(R)$, where $\gamma(t)$ is a unit speed geodesic with $\gamma(0)=e$ and $\gamma^{\prime}(0)=X_{0}+Z_{0}$. Then, Gaussian curvature $K(p)$ in $S_{e}(R)$ is given as follows:

$$
\begin{aligned}
K(p)= & \left(-\frac{1}{4}+\frac{\left|Z_{0}\right|^{2}\left(1-\left(1-\left|Z_{0}\right|^{2}\right) \cos \left(\left|Z_{0}\right| R\right)\right)}{2\left(1-\cos \left(\left|Z_{0}\right| R\right)\right)-\left(1-\left|Z_{0}\right|^{2}\right)\left|Z_{0}\right| R \sin \left(\left|Z_{0}\right| R\right)}\right) \\
& \times\left(-\frac{1}{4}\left(\left|Z_{0}\right|^{4}-\left|Z_{0}\right|^{2}+1\right)+\frac{\left|Z_{0}\right|^{2}}{2\left(1-\cos \left(\left|Z_{0}\right| R\right)\right)}\right)^{n-1} .
\end{aligned}
$$

Proof. By Proposition 3.3, we know that

for eack $k=1,2, \ldots, n$.

$$
\left[\begin{array}{c}
J_{2 k-1}(t) \\
J_{2 k}(t)
\end{array}\right]=B_{k}(t)\left[\begin{array}{c}
e_{2 k-1}(t) \\
e_{2 k}(t)
\end{array}\right]
$$


Since

$$
\left[\begin{array}{c}
J_{2 k-1}^{\prime}(t) \\
J_{2 k}^{\prime}(t)
\end{array}\right]=B_{k}^{\prime}(t)\left[\begin{array}{c}
e_{2 k-1}(t) \\
e_{2 k}(t)
\end{array}\right]+B_{k}(t)\left[\begin{array}{c}
e_{2 k-1}^{\prime}(t) \\
e_{2 k}^{\prime}(t)
\end{array}\right]
$$

by Lemma 3.2, we have that

$$
\begin{aligned}
{\left[\begin{array}{l}
J_{1}^{\prime}(t) \\
J_{2}^{\prime}(t)
\end{array}\right] } & =B_{1}^{\prime}(t)\left[\begin{array}{l}
e_{1}(t) \\
e_{2}(t)
\end{array}\right]+B_{1}(t)\left[\begin{array}{l}
e_{1}^{\prime}(t) \\
e_{2}^{\prime}(t)
\end{array}\right] \\
& =\left(B_{1}^{\prime}(t)+\frac{1}{2} B_{1}(t)\left[\begin{array}{cc}
0 & 1 \\
-1 & 0
\end{array}\right]\right)\left[\begin{array}{l}
e_{1}(t) \\
e_{2}(t)
\end{array}\right]
\end{aligned}
$$

and

$$
\begin{aligned}
{\left[\begin{array}{c}
J_{2 k-1}^{\prime}(t) \\
J_{2 k}^{\prime}(t)
\end{array}\right] } & =B_{k}^{\prime}(t)\left[\begin{array}{c}
e_{2 k-1}(t) \\
e_{2 k}(t)
\end{array}\right]+B_{k}(t)\left[\begin{array}{c}
e_{2 k-1}^{\prime}(t) \\
e_{2 k}^{\prime}(t)
\end{array}\right] \\
& =\left(B_{k}^{\prime}(t)+\frac{\left|Z_{0}\right|}{2} B_{k}(t)\left[\begin{array}{cc}
0 & 1 \\
-1 & 0
\end{array}\right]\right)\left[\begin{array}{c}
e_{2 k-1}(t) \\
e_{2 k}(t)
\end{array}\right]
\end{aligned}
$$

for each $k=2,3, \ldots, n$.

Since

$$
\left[\begin{array}{c}
e_{2 k-1}(t) \\
e_{2 k}(t)
\end{array}\right]=B_{k}(t)^{-1}\left[\begin{array}{c}
J_{2 k-1}(t) \\
J_{2 k}(t)
\end{array}\right]
$$

for eack $k=1,2, \ldots, n$, we have that

$$
\left[\begin{array}{l}
J_{1}^{\prime}(t) \\
J_{2}^{\prime}(t)
\end{array}\right]=\left(B_{1}^{\prime}(t)+\frac{1}{2} B_{1}(t)\left[\begin{array}{cc}
0 & 1 \\
-1 & 0
\end{array}\right]\right) B_{1}(t)^{-1}\left[\begin{array}{l}
J_{1}(t) \\
J_{2}(t)
\end{array}\right]
$$

and

$$
\left[\begin{array}{c}
J_{2 k-1}^{\prime}(t) \\
J_{2 k}^{\prime}(t)
\end{array}\right]=\left(B_{k}^{\prime}(t)+\frac{\left|Z_{0}\right|}{2} B_{k}(t)\left[\begin{array}{cc}
0 & 1 \\
-1 & 0
\end{array}\right]\right) B_{k}(t)^{-1}\left[\begin{array}{c}
J_{2 k-1}(t) \\
J_{2 k}(t)
\end{array}\right]
$$

for each $k=2,3, \ldots, n$.

Since the shape operator $S_{\gamma(t)}$ of the geodesic sphere $S_{e}(t)$ is given by

$$
S_{\gamma(t)}(J(t))=-J^{\prime}(t),
$$

the Gaussian curvature $K_{\gamma(t)}$ is

$$
\begin{aligned}
& K_{\gamma(t)} \\
= & \operatorname{det}\left(S_{\gamma(t)}\right) \\
= & \frac{\operatorname{det}\left(B_{1}^{\prime}(t)+\frac{1}{2} B_{1}(t)\left[\begin{array}{cc}
0 & 1 \\
-1 & 0
\end{array}\right]\right)}{\operatorname{det}\left(B_{1}(t)\right)} \times \prod_{k=2}^{n} \frac{\operatorname{det}\left(B_{k}^{\prime}(t)+\frac{\left|Z_{0}\right|}{2} B_{k}(t)\left[\begin{array}{cc}
0 & 1 \\
-1 & 0
\end{array}\right]\right)}{\operatorname{det}\left(B_{k}(t)\right)} .
\end{aligned}
$$

Direct calculations give that

$\frac{\operatorname{det}\left(B_{1}^{\prime}(t)+\frac{1}{2} B_{1}(t)\left[\begin{array}{cc}0 & 1 \\ -1 & 0\end{array}\right]\right)}{\operatorname{det}\left(B_{1}(t)\right)}=-\frac{1}{4}+\frac{\left|Z_{0}\right|^{2}\left(1-\left(1-\left|Z_{0}\right|^{2}\right) \cos \left(\left|Z_{0}\right| t\right)\right)}{2\left(1-\cos \left(\left|Z_{0}\right| t\right)\right)-\left(1-\left|Z_{0}\right|^{2}\right)\left|Z_{0}\right| t \sin \left(\left|Z_{0}\right| t\right)}$ 
and

$$
\frac{\operatorname{det}\left(B_{k}^{\prime}(t)+\frac{\left|Z_{0}\right|}{2} B_{k}(t)\left[\begin{array}{cc}
0 & 1 \\
-1 & 0
\end{array}\right]\right)}{\operatorname{det}\left(B_{k}(t)\right)}=-\frac{1}{4}\left(\left|Z_{0}\right|^{4}-\left|Z_{0}\right|^{2}+1\right)+\frac{\left|Z_{0}\right|^{2}}{2\left(1-\cos \left(\left|Z_{0}\right| t\right)\right)} .
$$

Hence, the Gaussian curvatue $K_{p}, p=\gamma(R)$ on the geodesic sphere $S_{e}(R)$ is given by

$$
\begin{aligned}
K(p)= & \left(-\frac{1}{4}+\frac{\left|Z_{0}\right|^{2}\left(1-\left(1-\left|Z_{0}\right|^{2}\right) \cos \left(\left|Z_{0}\right| R\right)\right)}{2\left(1-\cos \left(\left|Z_{0}\right| R\right)\right)-\left(1-\left|Z_{0}\right|^{2}\right)\left|Z_{0}\right| R \sin \left(\left|Z_{0}\right| R\right)}\right) \\
& \times\left(-\frac{1}{4}\left(\left|Z_{0}\right|^{4}-\left|Z_{0}\right|^{2}+1\right)+\frac{\left|Z_{0}\right|^{2}}{2\left(1-\cos \left(\left|Z_{0}\right| R\right)\right)}\right)^{n-1} .
\end{aligned}
$$

By Lemma 3.5, we have that

Corollary $3.11([9])$. Let $0<R<2 \pi$ and $S_{e}(R)$ be the geodesic sphere with center $e$ and radius $R$ in $\mathbb{H}^{3}$. Let $p=\gamma(R) \in S_{e}(R)$, where $\gamma(t)$ is a unit speed geodesic with $\gamma(0)=e$ and $\gamma^{\prime}(0)=X_{0}+Z_{0}$. Then, Gaussian curvature $K(p)$ of $S_{e}(R)$ is given as follows:

$$
K(p)=-\frac{1}{4}+\frac{\left|Z_{0}\right|^{2}\left(1-\left(1-\left|Z_{0}\right|^{2}\right) \cos \left(\left|Z_{0}\right| R\right)\right)}{2\left(1-\cos \left(\left|Z_{0}\right| R\right)\right)-\left(1-\left|Z_{0}\right|^{2}\right)\left|Z_{0}\right| R \sin \left(\left|Z_{0}\right| R\right)} .
$$

In particular, the Gaussian curvatures of the geodesic spheres on the 3-dimensional Heisenberg groups are greater than $-\frac{1}{4}$.

Remark 3.12. If $\left|Z_{0}\right|=1$, then $K_{p}=\left(-\frac{1}{4}+\frac{1}{2(1-\cos R)}\right)^{n}$. So, we see that $K_{p}$ goes to $\infty$ if the radius $R$ goes to $2 \pi$. And if $\left|Z_{0}\right|=0$, then since

$\lim _{\left|Z_{0}\right| \rightarrow 0}\left(-\frac{1}{4}+\frac{\left|Z_{0}\right|^{2}\left(1-\left(1-\left|Z_{0}\right|^{2}\right) \cos \left(\left|Z_{0}\right| R\right)\right)}{2\left(1-\cos \left(\left|Z_{0}\right| R\right)\right)-\left(1-\left|Z_{0}\right|^{2}\right)\left|Z_{0}\right| R \sin \left(\left|Z_{0}\right| R\right)}\right)=-\frac{1}{4}+\frac{6\left(2+R^{2}\right)}{r^{2}\left(12+R^{2}\right)}$ and

we see that

$$
\lim _{\left|Z_{0}\right| \rightarrow 0} \frac{\left|Z_{0}\right|^{2}}{2\left(1-\cos \left(\left|Z_{0}\right| R\right)\right)}=\frac{1}{R^{2}}
$$

$$
K_{p}=\left(-\frac{1}{4}+\frac{6\left(2+R^{2}\right)}{R^{2}\left(12+R^{2}\right)}\right)\left(-\frac{1}{4}+\frac{1}{R^{2}}\right)^{n-1}
$$

\section{References}

[1] J. Berndt, F. Tricerri, and L. Vanhecke, Geometry of generalized Heisenberg groups and their Damak-Ricci harmonic extensions, Lecture Notes in Mathematics, Vol. 1598, (1995), 51-68.

[2] M. P. do Carmo, Riemannian Geometry, Birkhauser, Boston, 1992.

[3] P. Eberlein, Geometry of 2-step Nilpotent Lie groups with a left invariant metric, Ann. Scient. Ecole Normale Sup. 27 (1994), 611-660.

[4] S. Gallot, D. Hulin, and J. Lafontaine, Riemannian Geometry, Springer-Verlag, Berlin, 1990. 
[5] C. Jang, J. Kim, Y. Kim, and K. Park, Conjugate points on the quaternionic Heisenberg group, J. Korean Math. Soc. 40 (2003), no. 1, 1-14.

[6] C. Jang and K. Park, Conjugate points on 2-step nilpotent groups, Geom. Dedicata 79 (2000), 65-80.

[7] A. Kaplan, Riemannian nilmanifolds attached to Clifford modules, Geom. Dedicata 11 (1981), 127-136.

[8] (1983), 35-42.

[9] R. Kim, On the geodesic spheres of the Heisenberg groups $\mathbb{H}^{3}$, Dissertations, University of Ulsan, 2003.

[10] M. S. Raghunathan, Discrete Subgroups of Lie Groups, Springer-Verlag, 1972.

[11] G. Walschap, Cut and conjugate Loci in two-step nilpotent Lie groups, Jour. of Geometric Analysis 7 (1997), 343-355.

[12] T. J. Wilmore, Riemannian Geometry, Oxford Univ. Press, 1993.

\author{
ChANGRIM JANG \\ Department of Mathematics \\ UNIVERSITY OF ULSAN \\ UlSAN 680-749, Korea \\ E-mail address: crjang@ulsan.ac.kr \\ JiHYe PARK \\ Myung-Duk Girl's Middle School \\ ILSANDONG DONGU \\ ULSAN 682-810, KoREA \\ E-mail address: click-79@hanmail.net \\ Keun Park \\ Department of Mathematics \\ UNIVERSITY OF ULSAN \\ ULSAN 680-749, Korea \\ E-mail address: kpark@ulsan.ac.kr
}

